\title{
A Second-Iteration Square Koch Fractal Slot Antenna for UHF Downlink Telemetry Applications in CubeSat Small Satellites
}

\author{
Jorge Simon (D), ${ }^{1}$ Jorge Flores-Troncoso, ${ }^{2}$ Jose Luis Alvarez-Flores, ${ }^{3}$ \\ Leonel Soriano-Equigua $\left(10,{ }^{3}\right.$ Marco Cardenas-Juarez, ${ }^{4}$ Armando Arce, ${ }^{5}$ Edgar Briones, ${ }^{6}$ \\ and Jorge Sosa-Pedroza ${ }^{7}$ \\ ${ }^{1}$ Cátedras CONACYT, Unidad Académica de Ingeniería Eléctrica, Universidad Autónoma de Zacatecas, Zacatecas, Mexico \\ ${ }^{2}$ Unidad Académica de Ingeniería Eléctrica, Universidad Autónoma de Zacatecas, Zacatecas, Mexico \\ ${ }^{3}$ Facultad de Ingeniería Mecánica y Eléctrica, Universidad de Colima, Colima, Mexico \\ ${ }^{4}$ Facultad de Ciencias, Universidad Autónoma de San Luis Potosí, San Luis Potosí, Mexico \\ ${ }^{5}$ Cátedras CONACYT, Facultad de Ciencias, Universidad Autónoma de San Luis Potosí, San Luis Potosí, Mexico \\ ${ }^{6}$ Instituto Tecnológico de Estudios Superiores de Occidente, Tlaquepaque, Mexico \\ ${ }^{7}$ Escuela Superior de Ingeniería Mecánica y Eléctrica, Instituto Politécnico Nacional, Mexico City, Mexico
}

Correspondence should be addressed to Jorge Simon; jsimonro@conacyt.mx

Received 11 June 2020; Accepted 17 September 2020; Published 9 October 2020

Academic Editor: Farid Ghanem

Copyright (C) 2020 Jorge Simon et al. This is an open access article distributed under the Creative Commons Attribution License, which permits unrestricted use, distribution, and reproduction in any medium, provided the original work is properly cited.

\begin{abstract}
This article presents the design, fabrication, and measurement of a square Koch fractal slot antenna for UHF band using both the FR4-G10 and Cuclad 250 substrates. Conveniently, this $56.56 \mathrm{~cm}$ full-length antenna possesses a geometry that allows it to be incorporated into the standardized $10 \mathrm{~cm} \times 10 \mathrm{~cm}$ faces of the CubeSats. Furthermore, it is shown that both selected substrates exhibit an acceptable performance at the frequency of interest despite the economic cost difference and relative permittivity. Hence, the commercial FR4-G10 antenna substrate can be preferred because of its low-cost and admissible performance at $458 \mathrm{MHz}$, which is a frequency in the UHF band that is commonly used for telemetry, tracking, and command downlinks of CubeSats. Measurements show that the proposed antenna exhibits a reflection coefficient of $-16.53 \mathrm{~dB}$, a bandwidth of $22.62 \mathrm{MHz}$ at $-10 \mathrm{~dB}$, a VSWR of 1.3508 , a normalized impedance of $0.794-j 0.173$ at $50 \Omega$, and a directivity of $2.24 \mathrm{dBi}$. The contribution of this work consists in the use of a fractal geometry to construct a low-cost slot antenna working at UHF frequencies over the limited area of the CubeSat faces and in order to optimize the area for an eventual coexistence with solar cells.
\end{abstract}

\section{Introduction}

The level of understanding that humanity currently has about the earth and the universe would not have been possible without the exploration of outer space by means of satellites [1]. Historically, scientific and technological advances due to the exploitation of knowledge of outer space have been subject to the drawbacks that large traditional satellites impose such as: (i) long development cycles, (ii) high construction and launch costs, (iii) congested frequency bands, and (iv) high economic risk in case of launch failure [2]. This has limited access to these resources to the most powerful economies on the planet, proportionally impacting their level of development. Recently, small satellite constellations have been envisioned as a means of achieving global connectivity at relatively low cost. Their possible applications in information systems range from aerial monitoring and land reconnaissance, prevention of natural or man-made disasters (e.g., earthquakes, floods, and forest fires), and connectivity between satellites, to advanced integrations that combine remote sensors in space and ground premises $[2,3]$. Thus, research efforts are oriented towards the development of small satellites that together offer the same benefits as traditional large satellites.

Within the classification of small satellites is the CubeSat, a standard characterized by having a mass of not more than $1.33 \mathrm{~kg}$ and maximum external dimensions of 
$10 \times 10 \times 10 \mathrm{~cm}^{3}$ for each unit (i.e., $\left.1 \mathrm{U}\right)$. Since its creation in 1999 [4, 5], hundreds of organizations worldwide have adopted the standard (either one unit or more), as it makes access to space affordable and relatively frequent, as there are more opportunities to carry a CubeSat in most launching vehicles [6]. Developers include not only universities and educational institutions but also private companies and government organizations [7].

Regardless of their size, satellites are made up of several subsystems, among which are (i) payload, (ii) on-board computer, (iii) power, (iv) communications, and (v) telemetry, tracking and command (TT\&C). As part of the latter are antenna and radio frequency (RF) modules, which allow communication between the satellites or between a satellite and a ground station to share sensor information, position, and control of the satellite [8].

In the context of satellite communication antennas, different types have been used for this purpose, among which are deployable, inflatable, and microstrip wire antennas [9]. Most of the small satellite antennas for VHF and UHF bands have been wire-type, as reported and marketed, respectively, in $[10,11]$, while small satellite printed type antennas have been mostly applied to the S-band [12], which are also commercially available [13]. Printed antennas have been booming due to their easy integration into communication systems and their high design flexibility [14]. These antennas are commonly manufactured using conventional printed circuit board (PCB) techniques and their characteristics make them excellent candidates for integration into small satellites. However, just few fractal printed antennas have been developed for CubeSat platforms; most of these works being focused on S-band frequencies, such as those developed in $[15,16]$, while fewer fractal designs for the UHF band have been reported like the one in [17], which is a microstrip second-iteration square Koch dipole antenna for TT\&C downlink applications in CubeSat satellites. That fractal antenna with a bandwidth of $50 \mathrm{MHz}$ was designed to work at $455 \mathrm{MHz}$, a frequency at which it had a maximum gain of $2.14 \mathrm{dBi}$.

Similarly, slot antennas that are often used at UHF and microwave frequencies are useful in many applications. Especially where flush or low-profile mounts are required, such as radars and sector antennas for cell phone base stations. These antennas consist of a flat metal plate with one or more holes or slots, which radiate electromagnetic waves similar to a dipole antenna when operating at a specific frequency [18]. Any slot has its complementary shape on wires and patches (microstrip), so the radiation pattern and impedance data for these shapes can be used to predict the patterns and impedances of the corresponding slots. This is largely based on a generalization and extension of the Babinet principle by Booker [19]. The main advantages of both slot antennas and patch antennas are their size, simplicity of design, and convenient adaptation as part of small satellite covers (CubeSats).

It is important to mention that the solar cells used to power the subsystems of a small satellite are located precisely on their covers and it is there where slot antennas can coexist, considering an adequate space optimization using
PCB technology, for example, Mahmoud in [20] integrated S-band antennas based on straight slots with solar panels for CubeSat satellites. So far, relatively few UHF printed fractal antennas have been reported and no reports of fractal slot antennas in this same band applied to small satellites have been found.

Table 1 shows a comparison of outstanding developments of on-board fractal antennas for small satellites that are reported in the recent literature (note that bandwidth is denoted as BW). It can be seen that fractal antennas for the $\mathrm{S}$-band predominate. In addition, bandwidths and gains are shown, which are some of the main design parameters.

Fractal geometries firstly studied by Mandelbrot [21] have found applications in the design of printed antennas for working at different frequencies and for diverse telecommunication standards or wireless services [22]. Examples of fractal antennas for ultrawide-band (UWB) applications are the one presented in [23] which is based on a fractal Koch geometry working from 3.1 to $10.6 \mathrm{GHz}$ and the one presented in [24] which is a modified Koch fractal antenna also for UWB applications, mainly for WLAN, Wimax, telemedicine, and other wireless applications. This article proposes the application of a fractal slot antenna on CubeSat faces, where it can share space with solar cells. The proposed antenna consists of a second-iteration square Koch fractal slot at UHF frequencies, frequencies that are commonly used for TT\&C downlinks. The contribution of this work consists in the use of a second-iteration square Koch fractal geometry to construct a slot antenna with a relatively large total length $(56.56 \mathrm{~cm})$ at UHF frequencies over a limited area of $10 \mathrm{~cm} \times 10 \mathrm{~cm}$, which is the area of the faces of a $1 \mathrm{U}$ CubeSat (where the solar cells are also placed). Conveniently, the antenna showed a single low significant reflection coefficient (acceptable impedance matching) in the band of interest (200 to $600 \mathrm{MHz}$ ).

The rest of the article is organized as follows: the proposed antenna is presented in Section 2; in Section 3, its performance is evaluated through simulations and measurements. The results are analyzed in Section 4, and finally, the conclusions are presented in Section 5.

\section{Materials and Methods}

2.1. Antenna Design. The antenna design consists of a fractal slot formed by two second-iteration square Koch curves (making an analogy with the two arms of a conventional dipole including the antenna feed). Each square Koch curve is obtained by repeatedly replacing each initial line segment by eight pieces, each piece is one quarter of the initial segment [25]. Koch curves, like other fractals, allow optimization of the total length of antennas [26], making possible to have antennas designed to operate at UHF frequencies on a relatively small face of a CubeSat. In this case, the slot antenna is built on the diagonal of a $10 \mathrm{~cm} \times 10 \mathrm{~cm}$ region. This region is, in turn, the central part of the two commercial laminates of $20.4 \mathrm{~cm} \times 25.4 \mathrm{~cm}$ used in manufacturing, which are FR4-G10 ${ }^{\circledR}(\mathrm{Cu}: 35 \mu \mathrm{m} / 1$ ozpsf) from Pulsar fx [27] and Cuclad $250^{\circledR}$ (Cu: $35 \mu \mathrm{m} / 1$ ozpsf) from Rogers Corporation [28], whose dielectric constants 
TABle 1: Comparison of some outstanding fractal antennas for CubeSats.

\begin{tabular}{lcccc}
\hline Reference & Type of antenna & Band & BW (MHz) & Gain (dBi) \\
\hline$[15]$ & Standing wave & S & 250 & 5.7 \\
{$[16]$} & Koch snowflake & S & 200 & 4.39 \\
{$[17]$} & Square Koch dipole & UHF & 50 & 1.565 \\
\hline
\end{tabular}

are 4.7 and 2.5, respectively. The thicknesses are $0.76 \mathrm{~mm}$ for FR4-G10 and $1.52 \mathrm{~mm}$ for Cuclad 250, respectively. For the Koch slot antenna, the total slot length of the nth iteration (2 second-iteration square Koch curves) is given by

$$
L_{\mathrm{TOT}}(n)=2\left(L_{n}\right)=2\left(2^{n} L_{0}\right)=2^{n+1} L_{0} \text {, }
$$

where $L_{n}$ denotes the length of each of the $2 n$th curves and $n$ is the integer number of iteration with $0 \leq n<\infty$ [25]. Thus, the second-iteration Koch slot antenna is located on the entire diagonal $D=2\left(L_{0}\right)=14.14 \mathrm{~cm}$ of a CubeSat face (an area of $10 \mathrm{~cm} \times 10 \mathrm{~cm}$ ) and has a total length $L_{\mathrm{TOT}}(2)=2$ $\left(L_{2}\right)=2^{2} D=56.56 \mathrm{~cm}$. As for the slot segments that make up the second-iteration Koch curve, they are $1.5 \mathrm{~mm}$ wide and $4.41 \mathrm{~mm}$ long.

The fractal slot antenna is coupled to a $50 \Omega$ SMA coaxial connector that acts as the power port. For example, in the case of $\lambda / 2$ slot antennas in a large sheet, the terminal resistance in the center of them is approximately $500 \Omega$ and the characteristic impedance of coaxial transmission lines is generally much minor $(50 \Omega$ or $75 \Omega)[19,29]$, so an offcenter feed is proposed to provide better impedance matching. In $\lambda / 2$ slots connected to a $50 \Omega$ coaxial cable, the distance from one end of the slot to the position of the feed port should be approximately $\lambda / 20$ [18]. In this design, after parametric studies, the antenna feed was placed $6.174 \mathrm{~cm}$ from one end of the slot, which according to the antenna work frequencies obtained by simulations and considering the effective dielectric constants for the FR4-G10 and Cuclad 250 substrates will be approximately $0.18 \lambda$ and $0.13 \lambda$, respectively. Figure 1 shows a schematic of the proposed antenna geometry as it would be located on the face of a $1 \mathrm{U}$ CubeSat $(10 \mathrm{~cm} \times 10 \mathrm{~cm})$, where it can be seen that diagonal length corresponds to $2 L_{0}=(0.02)^{0.5} \mathrm{~m}=14.14 \mathrm{~cm}$ and represents the total antenna length for the 0th iteration, while $4 L_{0}=28.28 \mathrm{~cm}$ for the 1 st iteration and $8 L_{0}=56.56 \mathrm{~cm}$ for the 2 nd one, respectively.

It is important to mention that slot antennas require an ideally infinite conductive plane [30]; this is not the case of the CubeSat standard with a limited area of $10 \mathrm{~cm} \times 10 \mathrm{~cm}$ on each face. Therefore, a larger conductor plane of $20.4 \mathrm{~cm} \times 25.4 \mathrm{~cm}$ is proposed. Although the dimensions are larger than the faces of a $1 \mathrm{U}$ CubeSat, this prototype could eventually be used for larger satellites. In the case of $1 \mathrm{U}$ CubeSats, the rest of the surface that does not correspond to the $10 \mathrm{~cm} \times 10 \mathrm{~cm}$ square could be flexible and unfolded after satellite launching.

Figure 2 shows a top view of the manufactured version of the slot antenna considering a $20.4 \mathrm{~cm} \times 25.4 \mathrm{~cm} \cdot \mathrm{PCB}$ laminate and the slot located in the central part $(10 \mathrm{~cm} \times 10 \mathrm{~cm})$ thereof.

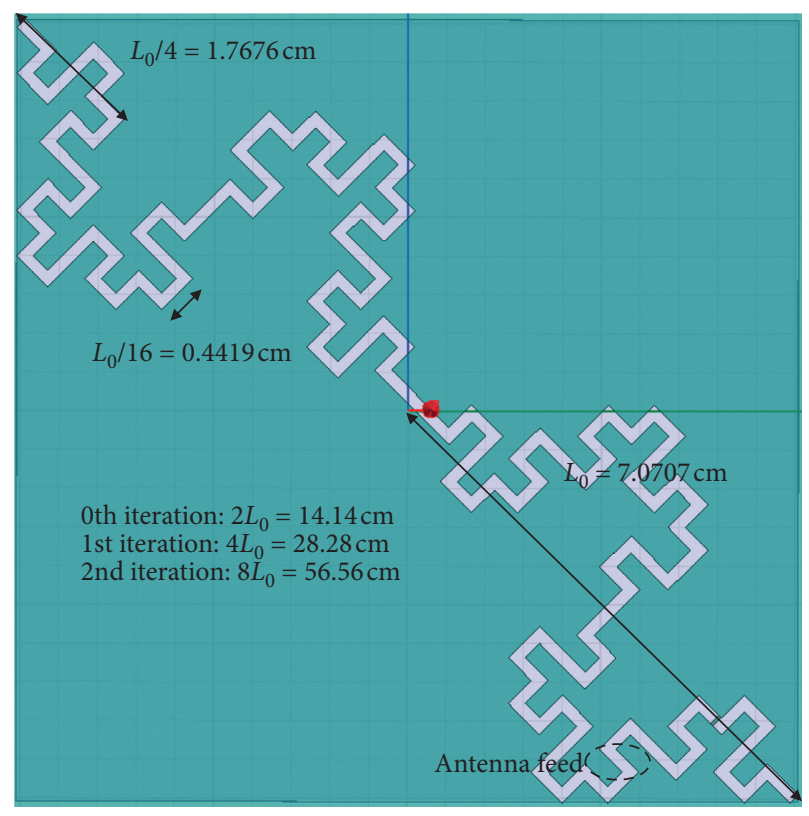

FIGURE 1: Schematic of the fractal slot antenna on a face of a $1 \mathrm{U}$ CubeSat satellite.

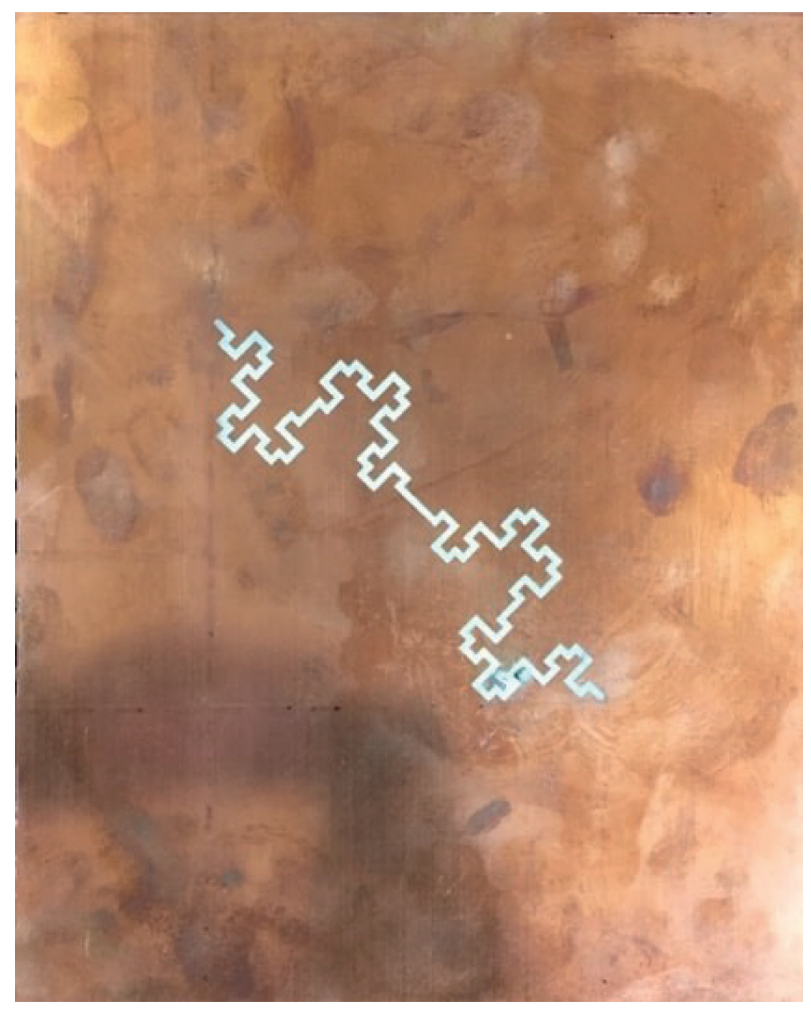

FIgUre 2: Top view of the implemented antenna $(20.4 \mathrm{~cm} \times 25.4 \mathrm{~cm})$.

2.2. Antenna Performance Evaluation. Computer simulations including reflection coefficient, voltage standing wave ratio, complex impedance, normalized electric field radiation patterns, surface current distribution, and axial ratio were performed to assess the antenna performance. 
Furthermore, with the intention of evaluating the actual performance of the antenna in a more realistic environment, the reflection coefficient, VSWR, and complex impedance were obtained using a vector network analyzer, and normalized electric field radiation patterns were obtained within an anechoic chamber located at the National Laboratory of Telecommunications and Antennas (LaNTA) belonging to the National Polytechnic Institute (IPN) of Mexico.

2.2.1. Simulations. For the computer evaluation of the proposed antenna, simulations based on the finite element method were performed. For this model, three regions were defined: a virtual radiation box (vacuum), an airbox (vacuum), and a box representing the substrates (FR4-G10 and Cuclad 250). Regarding sheets, two were defined: a rectangle for the lumped port and a sheet representing the ground plane (conductive plane surrounding the slot). For the airbox, a radiation boundary condition was considered, while for the ground plane, perfect-E boundary condition was considered. An excitation was considered in the lumped port in which impedance was set to $50 \Omega$. Respecting the mesh, it is worth mentioning that curved lines were configured. In addition, with respect to the frequency sweep, 201 linearly-spaced values from 200 to $600 \mathrm{MHz}$ were considered to evaluate the reflection coefficient $S_{11}$ in $\mathrm{dB}$ and VSWR for the two slot antennas to be manufactured using FR4-G10 and Cuclad 250 as substrates. In order to show the simulation environment used in the present work, a $3 \mathrm{D}$ view in Figure 3 is presented.

The parameter $S_{11}$ is a negative amount in $\mathrm{dB}$ that represents the power reflection towards the transmitter and it is also a measure of the degree of impedance matching between the transmitter and the antenna, which is given by

$$
S_{11}(\mathrm{~dB})=20 \log _{10}\left|S_{11}\right|=20 \log _{10}\left(\frac{\left|V_{\text {reflected }}\right|}{\left|V_{\text {forward }}\right|}\right),
$$

being

$$
S_{11}=\frac{Z_{A}-Z_{0}}{Z_{A}-Z_{0}}
$$

where $\left|V_{\text {reflected }}\right|$ is the reflected voltage toward the transmitter, $\left|V_{\text {forward }}\right|$ is the incident voltage toward the antenna, $Z_{A}=R_{A}+j X_{A}$ denotes the impedance of the load, which in this case is the antenna, and $Z_{0}$ is the impedance connected to it. Thus, the lower the value of $S_{11}$, the lower the power reflection towards the transmitter, and at the same time, a better impedance matching exists, fulfilling the goal of the antenna which is to radiate the maximum amount of energy into free space.

Finally, using the same frequency range used to plot $S_{11}$, voltage standing wave ratio VSWR and complex impedance were also computed. VSWR is written in terms of the reflection coefficient as follows [14]:

$$
\operatorname{VSWR}=\frac{1+\left|S_{11}\right|}{1-\left|S_{11}\right|}
$$

Likewise, by means of simulations, the normalized total electric field radiation patterns $\vec{E}_{n T}$ were obtained in order to know the antenna behavior in terms of far-field radiation, which is related to the magnitude of the simulated surface current distribution $\left|\vec{J}_{\text {surf }}\right|$. It is known that the vector potential $\vec{A}$ is related to the electric current $\vec{J}$ by the inhomogeneous Helmholtz equation (inhomogeneous vector wave equation):

$$
\nabla^{2} \vec{A}+k^{2} \vec{A}=-\mu \vec{J}
$$

being

$$
\vec{A}=\frac{\mu}{4 \pi} \iint_{S} \vec{J}_{s}\left(x^{\prime}, y^{\prime}, z^{\prime}\right) \frac{e^{-j k R}}{R} \mathrm{~d} S^{\prime},
$$

when $\vec{J}$ represents linear densities $\mathrm{m}^{-1}$.

Once $\vec{A}$ is known, the electric $\vec{E}$ and magnetic $\vec{H}$ farfields radiated by antennas of finite dimensions which are spherical waves are reduced and approximated to

$$
\vec{E} \simeq-j \omega \vec{A}
$$

and

$$
\vec{H} \simeq \frac{-j \omega}{\eta} \widehat{a_{r}} \times \vec{A}
$$

where $\vec{A}=\widehat{a_{r}} A_{r}(r, \theta, \varphi)+\widehat{a_{\theta}} A_{\theta}(r, \theta, \varphi)+\widehat{a_{\varphi}} A_{\varphi}(r, \theta, \varphi)[14]$.

2.2.2. Measurements. The antenna was characterized in terms of parameter $S_{11}$, VSWR, and its radiation patterns at frequencies in the band of interest which is UHF. The $S_{11}$ parameter and VSWR were obtained using a Keysight Technologies N5222A two-port vector network analyzer@ [31]. The configuration of the $S_{11}$ and VSWR measurements for the antennas made with the FR4-G10 and Cuclad 250 substrates is performed using the same parameters as the simulations. In this context, it is important to note that the FR4-G10 substrate is a lower cost material than the Cuclad 250 and therefore its performance is less robust. However, as it will be seen in the results section, the measurements of $S_{11}$ show that the FR4-G10 exhibits acceptable performance just like Cuclad 250, thus offering a good compromise considering the cost/benefit ratio. Likewise, normalized total electric field radiation patterns $\vec{E}_{n T}$ for the FR4-G10 antenna were obtained within an anechoic chamber located at the National Laboratory of Telecommunications and Antennas (LaNTA) belonging to the National Polytechnic Institute (IPN) of Mexico. This anechoic chamber uses a cylindrical antenna measurement system and delivers normalized electric field values of $\vec{E}_{n T}$. It should be noted that the LaNTA has ISO 9001:2015 certification no. SG20162448 "Antenna Calibration" and is accredited as "Calibration Laboratory" by the Mexican Accreditation Entity (EMA) with ACR-01 registration [32]. Figure 4 shows the antenna when measured inside the anechoic chamber.

Regarding the aforementioned measurements of radiation patterns, it is worth mentioning that in cylindrical coordinates measurement systems $(\rho, \varphi, z)$, the tangential 
components of the electric field radiated by the antenna under test (AUT) can be represented on the scanning cylinder as a superposition of elementary cylindrical waves [33]:

$$
\begin{aligned}
& E_{\varphi}(\varphi, z)=\sum_{n=-\infty}^{\infty} \int_{-\infty}^{\infty}\left[b_{n}(\eta) \frac{n \eta}{k d} H_{n}^{(2)}(\Lambda d)=-\left.a_{n}(\eta) \frac{\partial}{\partial \rho} H_{n}^{(2)}(\Lambda \rho)\right|_{\rho=d}\right] e^{j n \varphi} e^{-j \eta z} \mathrm{~d} \eta, \\
& E_{z}(\varphi, z)=\sum_{n=-\infty}^{\infty} \int_{-\infty}^{\infty} b_{n}(\eta) \frac{\Lambda^{2}}{k} H_{n}^{(2)}(\Lambda d) e^{j n \varphi} e^{-j \eta z} \mathrm{~d} \eta,
\end{aligned}
$$

where $a_{n}$ and $b_{n}$ are the cylindrical modal expansion coefficients:

$$
\begin{array}{r}
b_{n}(\eta) \frac{\Lambda^{2}}{k} H_{n}^{(2)}(\Lambda d)=\frac{1}{4 \pi^{2}} \int_{-\infty}^{\infty} \int_{-\pi}^{\pi} E_{z}(\varphi, z) e^{-j n \varphi} e^{j \eta z} \mathrm{~d} \varphi \mathrm{d} z, \\
b_{n}(\eta) \frac{n \eta}{k d} H_{n}^{(2)}(\Lambda d)-\left.a_{n}(\eta) \frac{\partial}{\partial \rho} H_{n}^{(2)}(\Lambda \rho)\right|_{\rho=d}=\frac{1}{4 \pi^{2}} \int_{-\infty}^{\infty} \int_{-\pi}^{\pi} E_{\varphi}(\varphi, z) e^{-j n \varphi} e^{j \eta z} \mathrm{~d} \varphi \mathrm{d} z,
\end{array}
$$

where $\Lambda=\sqrt{k^{2}-\eta^{2}}$.

Therefore, the electric field radiation patterns (far-field) in spherical coordinates are computed from the measured tangential components of the electric near-field over the square section cylinder. These far-field patterns are defined as [34]

$$
\begin{aligned}
& E_{\theta}(r, \theta, \varphi)=-j 2 k \sin (\theta) \frac{e^{-j k r}}{r} \sum_{n=-\infty}^{\infty} j^{n} b_{n} k \cos (\theta) e^{j n \varphi}, \\
& E_{\varphi}(r, \theta, \varphi)=-2 k \sin (\theta) \frac{e^{-j k r}}{r} \sum_{n=-\infty}^{\infty} j^{n} a_{n} k \cos (\theta) e^{j n \varphi}
\end{aligned}
$$

Once $E_{\theta}(r, \theta, \varphi)$ and $E_{\varphi}(r, \theta, \varphi)$ are known, the normalized total electric field for the main antenna planes can be obtained. Consequently, as it is well known axial ratio (AR) is the relationship between the major and minor axes of an ellipse representation of the electric field in a cycle of time. $\mathrm{AR}$ is a measure of field polarization, being generally elliptical and considered linear when AR tends to infinity. AR is defined as follows [14]:

$$
\mathrm{AR}(\mathrm{dB})=20 \log _{10}|\mathrm{AR}|=20 \log _{10}\left|\frac{\mathrm{OA}}{\mathrm{OB}}\right|,
$$

where $\mathrm{OA}$ is the magnitude of the mayor axis and $\mathrm{OB}$ is the magnitude of the minor axis.

\section{Results and Discussion}

This section presents results of simulations for $S_{11}$, bandwidth, VSWR, complex impedance, normalized total electric field radiation patterns, surface current distribution, and axial ratio, as well as measurements for $S_{11}$, bandwidth, VSWR, complex impedance, and normalized total electric field radiation patterns of the proposed fractal slot antenna for TT\&C applications on CubeSat satellites.

In Figure 5, a comparison of the $S_{11}$ parameter is shown in order to observe the effects of selected materials as antenna substrates for both simulations and measurements.

By means of simulations, an only impedance match frequency for the FR4-G10 substrate is observed between 200 and $600 \mathrm{MHz}$ at $403.2 \mathrm{MHz}$, with values of $S_{11}$ of $-10.22 \mathrm{~dB}$, a bandwidth at $-10 \mathrm{~dB}$ of $4.34 \mathrm{MHz}$, and a VSWR of 1.8905. Regarding simulations for the Cuclad 250 substrate, also an only impedance match frequency is observed between 200 and $600 \mathrm{MHz}$ at $448 \mathrm{MHz}$, with values of $S_{11}$ of $-15.21 \mathrm{~dB}$, a bandwidth at $-10 \mathrm{~dB}$ of $19.52 \mathrm{MHz}$, and a VSWR of 1.4196 . Even beyond $600 \mathrm{MHz}$, up to $1500 \mathrm{MHz}$, by simulations for both substrates, values for $S_{11}$ were not observed below $-10 \mathrm{~dB}$; the minimum observed values were greater than $-6 \mathrm{~dB}$. By measurements, also an only impedance match frequency for the FR4-G10 substrate is observed between 200 and $600 \mathrm{MHz}$ at $458 \mathrm{MHz}$, with values of $S_{11}$ of $-16.53 \mathrm{~dB}$, a bandwidth at $-10 \mathrm{~dB}$ of $22.62 \mathrm{MHz}$, and a VSWR of 1.3508. Measurements with the Cuclad 250 substrate showed an only impedance match frequency between 200 and $600 \mathrm{MHz}$ at $482 \mathrm{MHz}$, with values of $S_{11}$ value of $-19.55 \mathrm{~dB}$, a bandwidth at $-10 \mathrm{~dB}$ of $27 \mathrm{MHz}$, and a VSWR of 1.2353 .

Figure 6 shows normalized total electric field radiation patterns $\overrightarrow{E_{n T}}$, obtained through simulations and measurements for the slot fractal antenna made with the substrate FR4-G10. In this case, priority was given to the FR4-G10 substrate because the CubeSat standard promotes the use of commercial off-the-shelf (COTS) components. This is based on the fact that results of $S_{11}$ are acceptable for both substrates, being the FR4-G10 substrate much less expensive than the Cuclad 250 substrate. The normalized electric field radiation patterns $\overrightarrow{E_{n T}}$ are shown for the horizontal diagram 


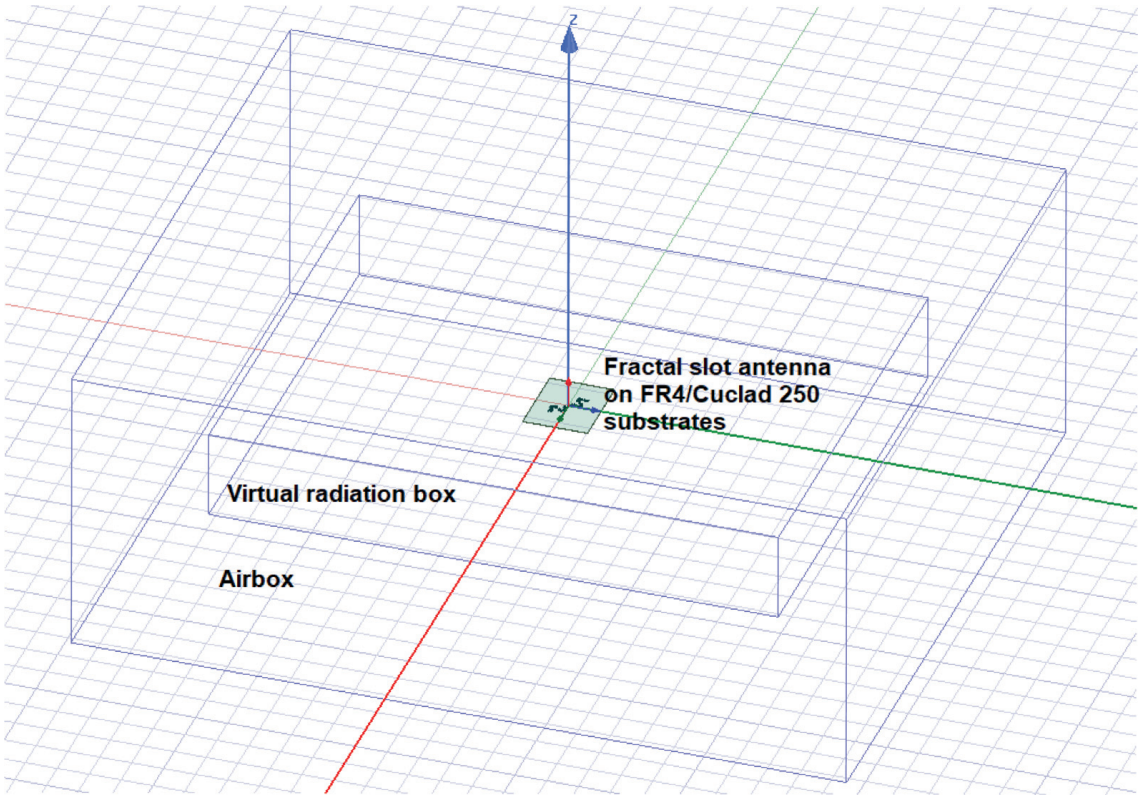

FIGURE 3: Simulation environment for the slot fractal antenna where the geometry can be observed including the subdomains considered.

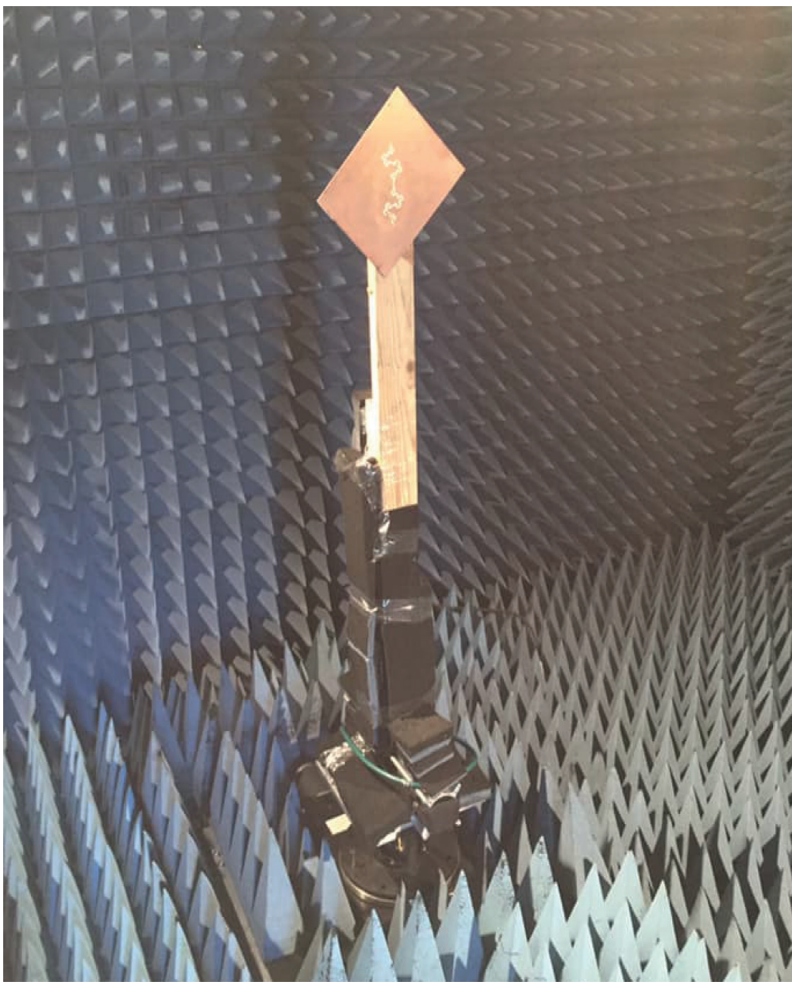

FIGURE 4: The second-iteration fractal slot antenna mounted when measured inside the anechoic chamber.

(HD) and the vertical diagram (VD), denoted for the simulations by the abbreviations SHD and SVD, respectively. Thus, for the FR4-G10 substrate antenna at the impedance match frequency observed at $403.2 \mathrm{MHz}$ by simulations, the approximate directivities $(D)$ were obtained using Pozar and McDonald equations [14], which yield values of $1.95 \mathrm{dBi}$ and $1.83 \mathrm{dBi}$, respectively. Additionally, the measured radiation patterns MHD and MVD at the impedance match frequency of $458 \mathrm{MHz}$ (observed on the $S_{11}$ measurement plot for FR4-G10) are shown, which were obtained using the anechoic chamber belonging to LaNTA. The cylindrical measurement system in the chamber rotates the base on which the antenna under test is mounted, which is separated from a rectangular waveguide probe that 


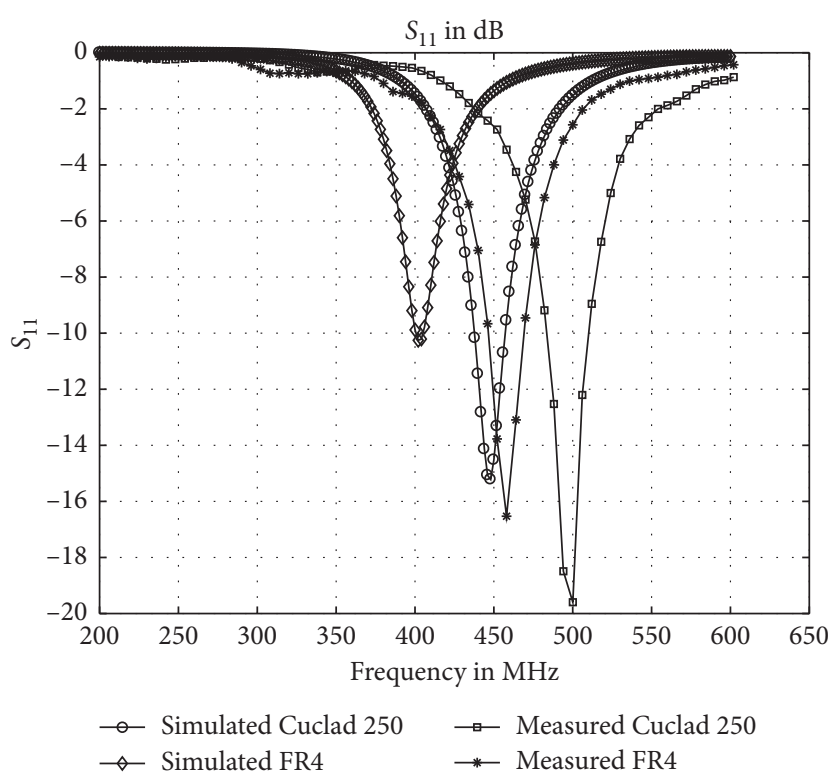

FIGURE 5: Comparison of reflection coefficient $S_{11}$ obtained through simulations and measurements vs frequency for the designed antenna using two different materials as substrates.

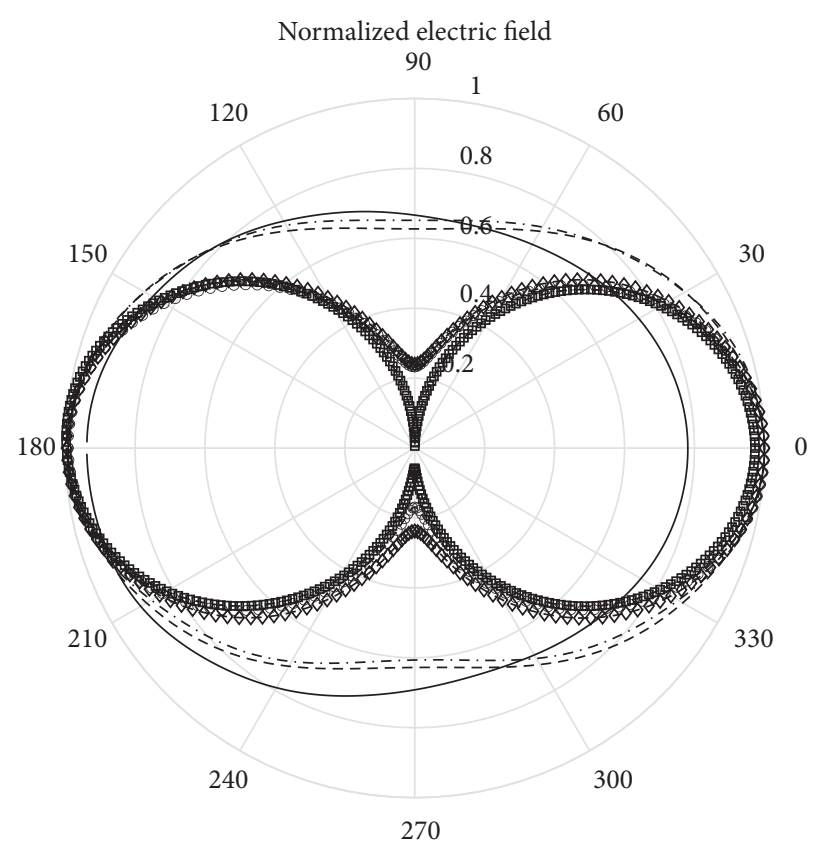

- Measured HD at $458 \mathrm{MHz}$

- $\because$ - Measured VD at $458 \mathrm{MHz}$

- - - Simulated HD at $403.2 \mathrm{MHz}$

$-\diamond-$ Simulated VD at $403.2 \mathrm{MHz}$

. . - Simulated HD at $458 \mathrm{MHz}$

$-\ominus$ - Simulated VD at $458 \mathrm{MHz}$

FIGURE 6: Comparison of normalized electric field radiation patterns obtained through simulations and measurements for the FR4G10 substrate where angles are in degrees.

performs an elevation scan. In this case, the approximate directivities $(D)$ were also obtained using the Pozar and McDonald equations, producing values of $2.24 \mathrm{dBi}$ and $2.10 \mathrm{dBi}$, respectively.
As a complementary result, the simulated HD and VD at $458 \mathrm{MHz}$, which is the impedance match frequency obtained in the measurements, are shown, noticing that no significant differences are observed with the HD and VD plots for the normalized electric field radiation patterns $\underset{E_{n T}}{\longrightarrow}$ obtained through measurements at $458 \mathrm{MHz}$.

Figure 7 shows the shapes of the 3D plots for the electric field radiation pattern measurements at $458 \mathrm{MHz}$ obtained inside the anechoic chamber, i.e., VD (left) and HD (right). $\mathrm{VD}$ and $\mathrm{HD}$ in $3 \mathrm{D}$ view show the omnidirectional behavior of the antenna proposed in this work, which is common in TT\&C on-board antennas for applications on small satellites.

In Figure 8, the simulated surface electric current distribution on the antenna surface at the impedance match frequency of 403.2 MHz for the FR4-G10 substrate and at the phase angle of $\omega t=0^{\circ}$ is showed, this is in order to observe the behavior and its relation with radiation patterns (see equation (5)) and with respect to polarization, where it can be noticed that the fractal antenna has transversal current elements interfering with the ones over the antenna axis ( $y$-axis). These transversal elements cause a slant of the E-field radiation pattern with respect to the antenna axis, which does not happen in straight slot antennas.

Finally, in Figure 9, the slant of the E-field can be observed, being the maximum axial ratio at $70^{\circ}$ with respect to the $x$-axis ( $20^{\circ}$ with respect to the $y$-axis), whose value of $10,935(80.77 \mathrm{~dB})$ means linear polarization in that direction.

In Table 2, the information regarding the proposed FR4G10 substrate slot antenna is synthesized, both for simulations and measurements, which leads to conclude that the proposed slot antenna that uses FR4-G10 as a substrate is suitable for use in multichannel transceivers commonly used in the assembly of TT\&C modules in small CubeSat satellites 

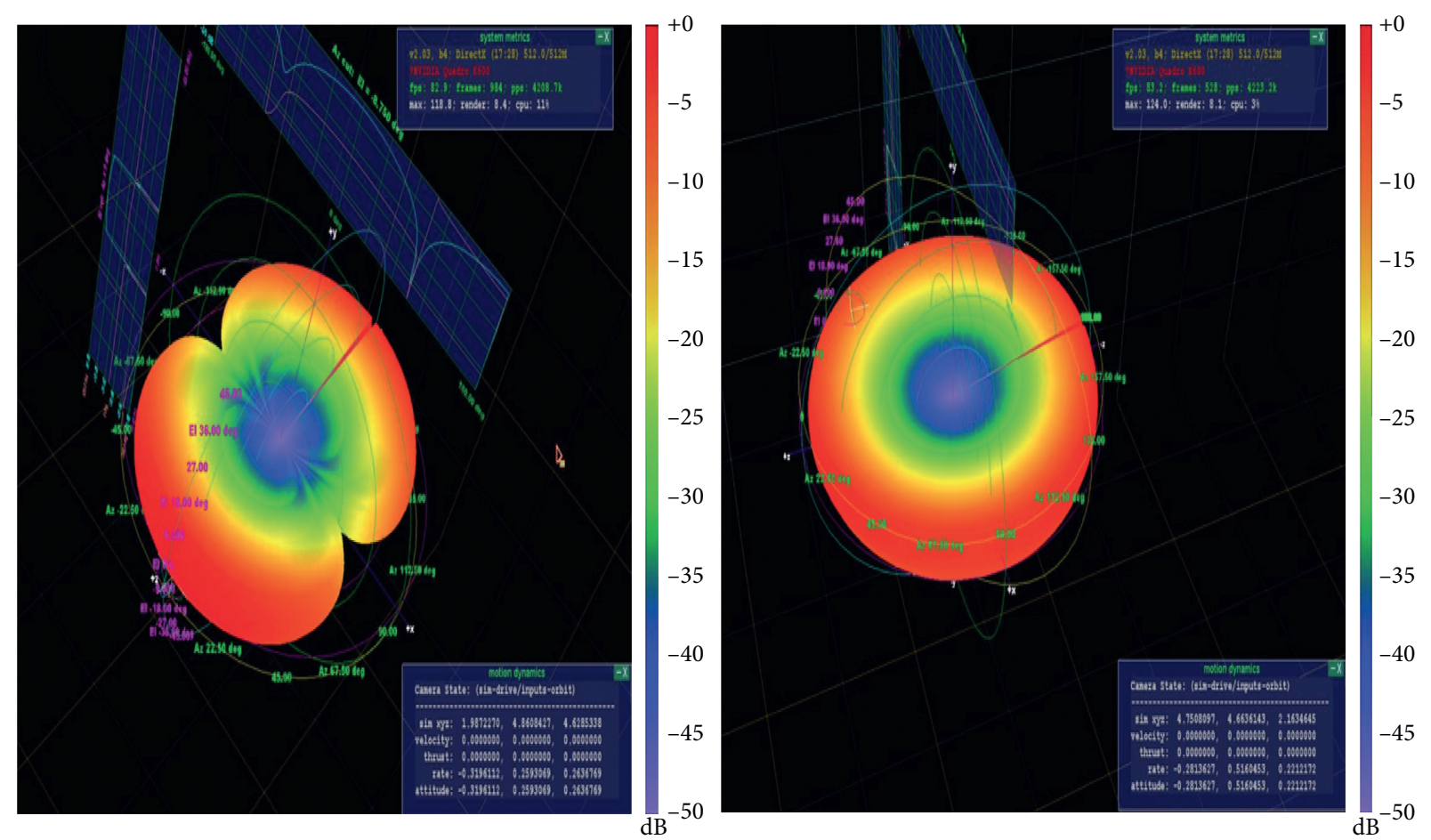

FIGURE 7: 3D plots for the electric field radiation pattern measurement at $458 \mathrm{MHz}$ inside an anechoic chamber considering the FR4-G10 substrate and corresponding to VD (left) and HD (right).

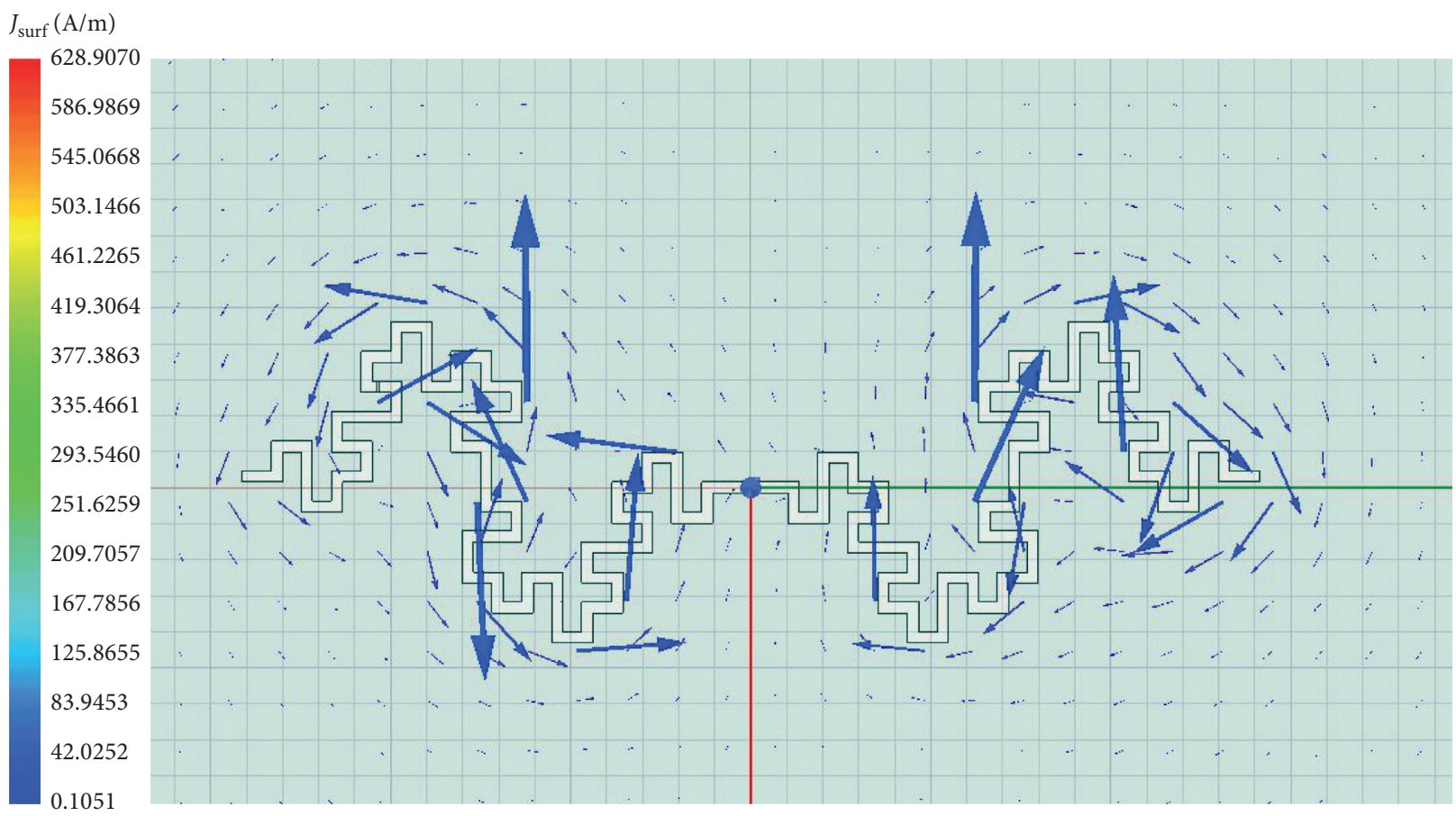

FIgURE 8: Simulated surface electric current distribution at phase angle $\omega t=0^{\circ}$.

[35]. Those multichannel transceivers operate at frequencies in the $458 \mathrm{MHz}$ band and within a narrow band of $(25 \mathrm{KHz})$, which is significantly surpassed by the slot antenna designed in this research, whose bandwidth at $-10 \mathrm{~dB}$ is $22.62 \mathrm{MHz}$.
The FR4-G10 antenna presented in this work is the slot version of the one purposed in [17], which was microstrip and made on bakelite. That microstrip version was centerfed, while the slot version presented in this work considered 


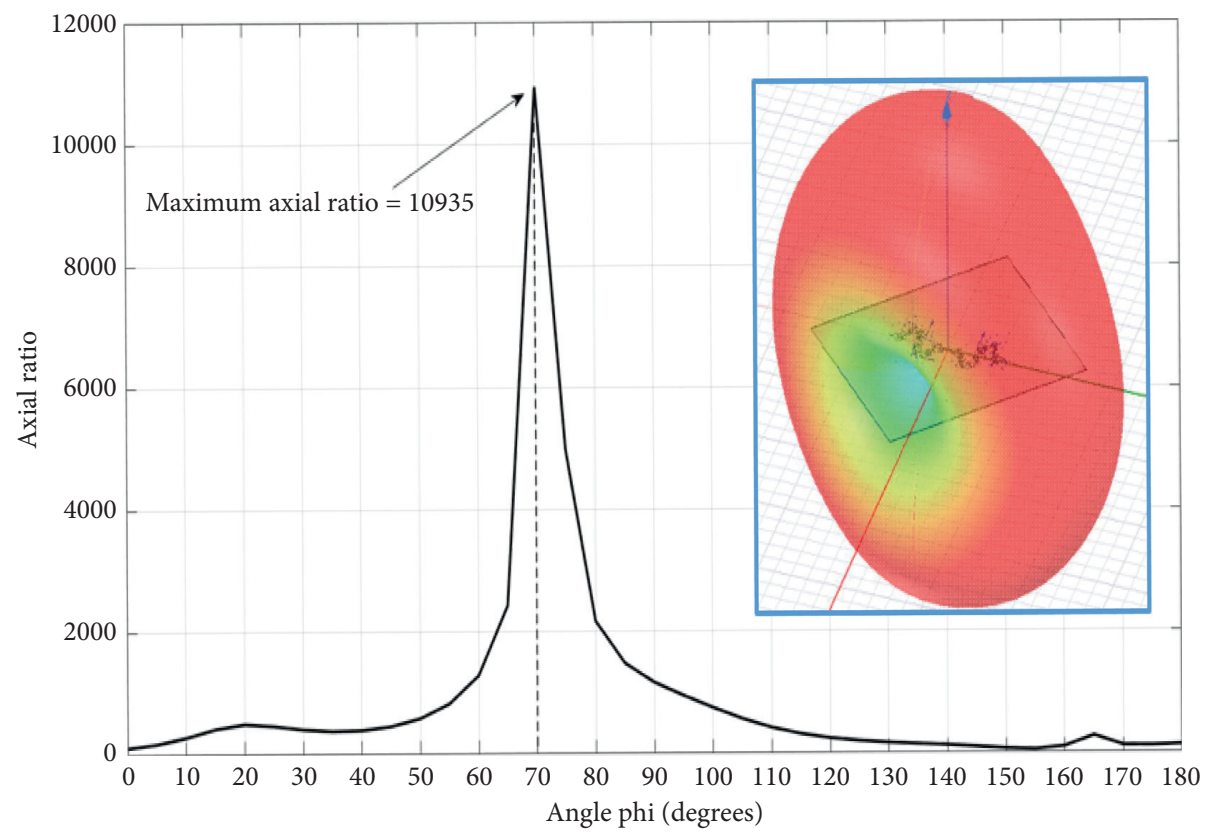

Figure 9: Simulated axial ratio at $\theta=90^{\circ}$.

TABLE 2: Comparison of parameters obtained by simulations and measurements for the proposed antenna on FR4-G10.

\begin{tabular}{lccccccc}
\hline Type of methodology & $\begin{array}{c}\text { Work } \\
\text { frequency }\end{array}$ & $S_{11}(\mathrm{~dB})$ & $\begin{array}{c}\text { BW at }-10 \mathrm{~dB} \\
(\mathrm{MHz})\end{array}$ & VSWR & $\begin{array}{c}\text { Normalized } \\
\text { impedance }(50 \Omega)\end{array}$ & D Pozar (dBi) & D McDonald (dBi) \\
\hline Simulation & 403.2 & -10.22 & 4.34 & 1.8905 & $0.7798-j 0.5257$ & 1.95 & 1.83 \\
Measurement & 458.0 & -16.53 & 22.62 & 1.3508 & $0.794-j 0.173$ & 2.24 & 2.10 \\
\hline
\end{tabular}

an off-center feed. The slot version in this work allows the eventual placement of solar cells, since this antenna is on the CubeSat faces where the solar cells could coexist as mentioned in [20]. Likewise, it is worth mentioning that although the FR4-G10 antenna has a maximum radiation efficiency of $76.13 \%$ at the simulation match frequency, while for Cuclad 250 antenna is $96.57 \%$, the performance for the low-cost FR4-G10 is still relatively acceptable in terms that the CubeSat standard encourages the use of COTS components.

\section{Conclusions}

In this article, the design, fabrication (FR4-G10 and Cuclad 250), and measurement of a second-iteration square Koch fractal slot antenna for application in TT\&C downlinks at UHF band frequencies for CubeSat small satellites are presented. The tests showed that the antenna performance in terms of parameter $S_{11}$ and VSWR is acceptable for both substrates, being preferred FR4-G10 for its low cost and acceptable radiation efficiency in accordance with the vocation of the CubeSat standard to use COTS components. At a frequency of $458 \mathrm{MHz}$, where narrowband UHF multichannel transceivers used on CubeSats are usually tuned, the FR4-G10 slot antenna showed satisfactory impedance matching. Results showed that measurement for parameter $S_{11}$ was $-16.53 \mathrm{~dB}$, with a bandwidth at $-10 \mathrm{~dB}$ of $22.62 \mathrm{MHz}$, a VSWR of 1.3508 , and a normalized impedance of $0.794-j 0.173$ at $50 \Omega$, while the directivity at this same frequency was $2.24 \mathrm{dBi}$, a typical directivity in the case of omnidirectional antennas. The total physical length of the antenna was $56.56 \mathrm{~cm}$, thus optimizing the diagonal length of $14.14 \mathrm{~cm}$ (0th iteration) of the CubeSat standard. The second iteration was chosen because it is the one that showed the longest total antenna length (lowest impedance match frequency) without resorting to the third iteration that could generate a match frequency outside the band of interest, having too small lengths of the elements that make up the fractal using the PCB technique. Furthermore, the designed antenna can be seamlessly coupled to commercial transceivers with an output impedance of $50 \Omega$.

\section{Data Availability}

The data supporting this research article are available upon request to the corresponding author.

\section{Conflicts of Interest}

The authors declare that there are no conflicts of interest regarding the publication of this paper.

\section{Acknowledgments}

This work has been partially supported by the Mexican $\mathrm{Na}$ tional Council for Science and Technology (CONACYT) through its CATEDRAS-CONACYT Program (Project 3066) and the Program for Professors' Development (PRODEP) 
from the Federal Mexican Government. The authors thank the National Laboratory of Telecommunications and Antennas (LANTA) for the facilities granted for the use of the anechoic chamber and the Autonomous University of Zacatecas (UAZ) for allowing the use of measurement instruments belonging to the Center for Research, Innovation, and Development in Telecommunications (CIDTE).

\section{References}

[1] M. N. Sweeting, "Modern small satellites-changing the economics of space," Proceedings of the IEEE, vol. 106, no. 3, pp. 343-361, 2018.

[2] I. F. Akyildiz and A. Kak, "The internet of space things/ CubeSats: a ubiquitous cyber-physical system for the connected world," Computer Networks, vol. 150, pp. 134-149, 2019.

[3] Y. Rahmat-Samii, V. Manohar, and J. M. Kovitz, "For satellites, think small, dream big: a review of recent antenna developments for CubeSats," IEEE Antennas and Propagation Magazine, vol. 59, no. 2, pp. 22-30, 2017.

[4] B. G. Evans, "Satelite Communication Systems," Institution of Engineering and Technology, London, UK, 2008.

[5] California Polytechnic State University, "The CubeSat program," https://www.cubesat.org/about.

[6] R. S. Jahku and J. Pelton, Small Satellites and Their Regulation, Springer, New York, NY, USA, 2014.

[7] NASA, "Small satellite missions," https://www.nasa.gov/ missionpages/smallsats.

[8] C. Kakoyiannis and P. Constantinou, "Electrically small microstrip antennas targeting miniaturized satellites: the CubeSat paradigm, microstrip antennas," in Microstrip Antennas, N. Nasimuddin, Ed., IntechOpen, London, UK, pp. 273-316, 2011.

[9] S. Gao, Y. Rahmat-Samii, R. E. Hodges, and X.-X. Yang, "Advanced antennas for small satellites," Proceedings of the IEEE, vol. 106, no. 3, pp. 391-403, 2018.

[10] K. S. Sadasivan, S. N. Shalini, B. S. Cheela, and N. Annavarapu, "Design and analysis of antennas for a nanosatellite," in Proceedings of the 2017 IEEE Aerospace Conference, pp. 1-9, Big Sky, MT, USA, March 2017.

[11] EnduroSat, "UHF antenna," https://www.endurosat.com/ cubesat-store/all-cubesat-modules/uhfantenna.

[12] F. E. Tubbal, R. Raad, K. Chin, and B. Butters, "S-band shorted patch antenna for inter pico satellite communications," in Proceedings of the 2014 8th International Conference on Telecommunication Systems Services and Applications (TSSA), pp. 1-4, Kuta, Indonesia, October 2014.

[13] EnduroSat, "S-Band antenna commercial," https://www.endurosat. com/cubesat-store/all-cubesat-modules/s-bandantenna-commercial.

[14] C. A. Balanis, Antenna Theory: Analysis and Design, Wiley, Hoboken, NJ, USA, 2005.

[15] C. A. Figueroa-Torres, J. L. Medina-Monroy, H. LobatoMorales, R. A. Chávez-Pérez, and A. Calvillo-Téllez, "A microstrip antenna based on a standing-wave fractal geometry for CubeSat applications," Microwave and Optical Technology Letters, vol. 58, no. 9, pp. 2210-2214, 2016.

[16] O. F. González-Palacios, R. E. Díaz Vargas, J. A. HeraudPerez, and S. B. Correa-Erazo, "S-band koch snowflake fractal antenna for CubeSats," in Proceedings of the 2016 IEEE ANDESCON, Arequipa, Peru, October 2016.

[17] J. Simón, J. L. Alvarez-Flores, J. Villanueva-Maldonado, V. H. Castillo-Topete, L. Soriano-Equigua, and J. Flores-
Troncoso, "A microstrip second-iteration square koch dipole antenna for TT\&C downlink applications in small satellites," International Journal of Antennas and Propagation, vol. 2017, Article ID 4825179, 8 pages, 2017.

[18] J. D. Kraus and R. J. Marhefka, Antennas for All Applications, McGraw-Hill Science/Engineering/Math, New York, NY, USA, 3rd edition, 2001.

[19] H. G. Booker, "Slot aerials and their relation to complementary wire aerials (Babinet's principle)," Journal of the Institution of Electrical Engineers-Part IIIA: Radiolocation, vol. 93, no. 4, pp. 620-626, 1946.

[20] M. N. Mahmoud, "Integrated solar panel antennas for CubeSat satellites," MS thesis, Utah State University, Logan, UT, USA, 2010.

[21] B. Mandelbrot, "The Fractal Geometry of Nature," W. H. Freeman and Company, New York, NY, USA, 1983.

[22] J. P. Gianvittorio and Y. Rahmat-Samii, "Fractal antennas: a novel antenna miniaturization technique, and applications," IEEE Antennas and Propagation Magazine, vol. 44, no. 1, pp. 20-36, 2002.

[23] F. B. Zarrabi, Z. Mansouri, N. P. Gandji, and H. Kuhestani, "Triple-notch UWB monopole antenna with fractal koch and T-shaped stub," AEU-International Journal of Electronics and Communications, vol. 70, no. 1, pp. 64-69, 2016.

[24] S. Rani and A. P. Singh, "Modified Koch fractal antenna with asymmetrical ground plane for multi and UWB applications," International Journal of Applied Electromagnetics and Mechanics, vol. 42, no. 2, pp. 259-267, 2012.

[25] A. Mondal, S. Chakraborty, R. K. Singh, and R. Ghatak, "Miniaturized and dual band hybrid koch fractal dipole antenna design," in Proceedings 2011 International Conference on Computer, Communication and Electrical Technology (ICCCET), pp. 193-197, Tamilnadu, India, March 2011.

[26] D. H. Werner and S. Ganguly, "An overview of fractal antenna engineering research," IEEE Antennas and Propagation Magazine, vol. 45, no. 1, pp. 38-57, 2003.

[27] Pulsar, "FR4-G10 laminates," https://pcbfx.com/mainsite/ pages/products/flexpcboard.html.

[28] Rogers Corporation, "Cuclad 217/233/250 laminates," https:// www.rogerscorp.com/acs/products/90/CuClad-217-233-250Laminates.aspx.

[29] C. Theofanopulus, "Performance evaluation of a half-wave resonant slot antenna over perfect ground," MS thesis, Naval Postgraduate School, Monterey, CA, USA, 1987.

[30] W. Chen, The Electrical Engineering Handbook, Academic Press, Burlington, MA, USA, 2005.

[31] Keysight Technologies, "PNA microwave network analyzer," https:// www.keysight.com/en/pdx-x201875-pn-N5222A/pnamicrowavenetwork-analyzer-265-ghz?cc=US\&lc=eng.

[32] Instituto Politénico Nacional (México), “The Mexican national laboratory for telecommunications and antennas (LaNTA)," https://www.ipn.mx/technopoli/serviciostecnol\% C3\%B3gicos-especializados.html.

[33] M. Farouq, M. Serhir, and D. Picard, "Matrix method for farfield calculation using irregular near-field samples for cylindrical and spherical scanning surfaces," Progress In Electromagnetics Research B, vol. 63, pp. 35-48, 2015.

[34] M. Farouq, M. Serhir, and D. Picard, "Far-field determination from near-field measured over a square section cylinder," in Proceedings of the 2016 IEEE Conference on Antenna Measurements \& Applications (CAMA), Syracuse, NY, USA, October 2016.

[35] Radiometrix, "UHF narrowband FM multichannel transceiver," https://www.radiometrix.com/node/187. 